(2) Open Access Full Text Article

\title{
Successful conjunctival socket expansion in anophthalmic patients until the age of 2 years: an outpatient procedure
}

This article was published in the following Dove Press journal:

Clinical Ophthalmology

12 September 2016

Number of times this article has been viewed

\author{
Rania Assem El Essawy \\ Sameh Hassan Abdelbaky \\ Department of Ophthalmology, \\ Faculty of Medicine, Cairo University, \\ Cairo, Egypt
}

Purpose: To report the results of a simple outpatient method for soft tissue socket expansion in young children with congenital anophthalmos.

Methods: Seventeen congenital anophthalmic sockets of 15 infants of a mean age of 4.2 \pm 4.4 months were fitted with specially designed serial solid acrylic shapes or hydrogel expanders using cyanoacrylate for eyelids closure when using the latter.

Results: At the age of 2 years, the mean horizontal eyelid length increased from a mean of $11.6 \pm 4.5$ to $19.4 \pm 4.6 \mathrm{~mm}$ and the volume of the last expander from a mean of $0.6 \pm 0.2$ to $2.0 \pm 0.3 \mathrm{~cm}^{3}$. The specially designed acrylic shapes could be a substitute to the custom-made molds, which require general anesthesia.

Conclusion: Successful increase in the horizontal eyelid length as well as the conjunctival socket volume could be achieved by a simple outpatient procedure without the need for repeated hospitalization and general anesthesia in these infants.

Keywords: congenital anophthalmia, custom-made conformers, hydrogel expanders, cyanoacrylate glue

\section{Introduction}

Congenital anophthalmos is one of the most frustrating conditions that may face the ophthalmologist especially if it is bilateral. It is a rare malformation with an incidence reported to be between 0.6 and 4.2 per 100,000 births $;{ }^{1}$ in this condition, there is a tiny orbit and tiny socket coexisting with tiny eyelids that are small both horizontally and vertically thus preventing the retention of a suitable prosthesis. The ideal management would be simultaneous expansion of the orbital bones with the socket and eyelids. Although there are no evidence-based studies to prove a correlation between the age at initial socket expansion and the outcome, it is a general consensus that it is important to start expanding the contracted socket as early as possible. This is supported by the fact that the growth of the affected orbit slows down or even stagnates by 3 years of age. ${ }^{2,3}$ Therefore, intervention therapy before 3 years of age is critical. Apart from few successful trials, ${ }^{4,5}$ early osteotomies before orbital expanders performed in an early age before 3 years have been mostly unsuccessful and frequently lead to retardation of orbital growth and overlying soft tissue contracture, as Wolfe commented back in 1989:6 "even in the best of circumstances, the results are poor". Therefore, most surgeons ${ }^{6-10}$ believe in the concept of "active conservative treatment" in which early external orbital conformers are used for socket expansion followed by a subsequent orbital surgery, if necessary, when the child becomes 3 or 4 years old.
Correspondence: Sameh Hassan Abdelbaky

Department of Ophthalmology, Faculty of Medicine, Cairo University, Abdelaziz al

Seoud st. Manial, Cairo, Egypt

Tel +002 0122106 I 228

Email samhassan76@gmail.com 
A number of methods have been employed for early socket expansion mainly mechanical or tension wire expanders, pressure conformers, and progressive-sized conformers. With the first two methods, overly aggressive pressure or tension expansion, often inducing harmful scarring by forcing the socket to conform to the shape of the pressure template, a condition termed "deformity by conformity", a gradual stepwise socket expansion by progressively-sized conformers has been favored. ${ }^{11}$ Currently, there are two preferred techniques of early socket expansion: the first involves the use of serial custom-made solid conformers formed according to serial socket molds, and the second is using hydrophilic expandable hydrogen polymers with tarsorrhaphy. Both techniques usually involve hospitalization and general anesthesia. The purpose of this work is to describe a simple method for soft tissue socket expansion in young children that can be employed in the outpatient clinic.

\section{Methods}

In this noncomparative case series, the data of 17 eyes of 15 infants (nine females and six males) with congenital clinical anophthalmia who underwent a staged conjunctival cavity expansion on an outpatient basis in the period between January 2009 and December 2013 were retrospectively reviewed. One of these infants had an associated congenital coloboma of the upper eyelid, and another three showed other facial anomalies in the form of hemifacial atrophy and/or cleft lip and cleft palate. Socket expansion was started earliest at 4 weeks and latest by 15 months of age (mean $4.2 \pm 4.4$ months). Gradual expansion was achieved by using serial solid acrylic prostheses specially designed by the authors or initially by self-inflating hydrophilic hydrogel expanders of $0.4,0.9$, and $1.5 \mathrm{~mL}$ final volumes (AcriTec; GmbH, Glienecke/Berlin, Germany) (Figure 1). The child was wrapped in a blanket, and horizontal eyelid measurements were taken. Topical anesthetic eye drops were applied while examining the socket determining its depth and shape. A socket expander whether solid or hydrogel, when available, of an appropriate size and shape in case of acrylic expanders was inserted and the eyelids were allowed to close gently. Thorough drying of the eyelid margin with a piece of gauze was followed by the application of cyanoacrylate glue (Histoacryl ${ }^{\circledR}$, TissueSeal LLC, Ann Arbor, MI, USA) on the lid margins (and not the eyelashes) to close the eyelids in case of hydrogel expanders, and the cyanoacrylate was allowed to dry completely. Topical antibiotic drops were prescribed for 2 weeks followed by a tear substitute eye drops. The child was reviewed every 2 weeks. In the first visit, the cyanoacrylate glue was brushed off the eyelids gently with gauze and warm water. The socket expander was increased in size when it starts to rotate within the orbit or when the eyelids become relatively loose around it and this was usually between 3 weeks and 2 months according to the age of the child (the younger, the earlier due to the faster rate of growth). This was repeated until at least the age of 2 years. Children who dropped follow-up before the age of 2 were excluded from the study. The degree of orbital expansion, at the age of 2 ,
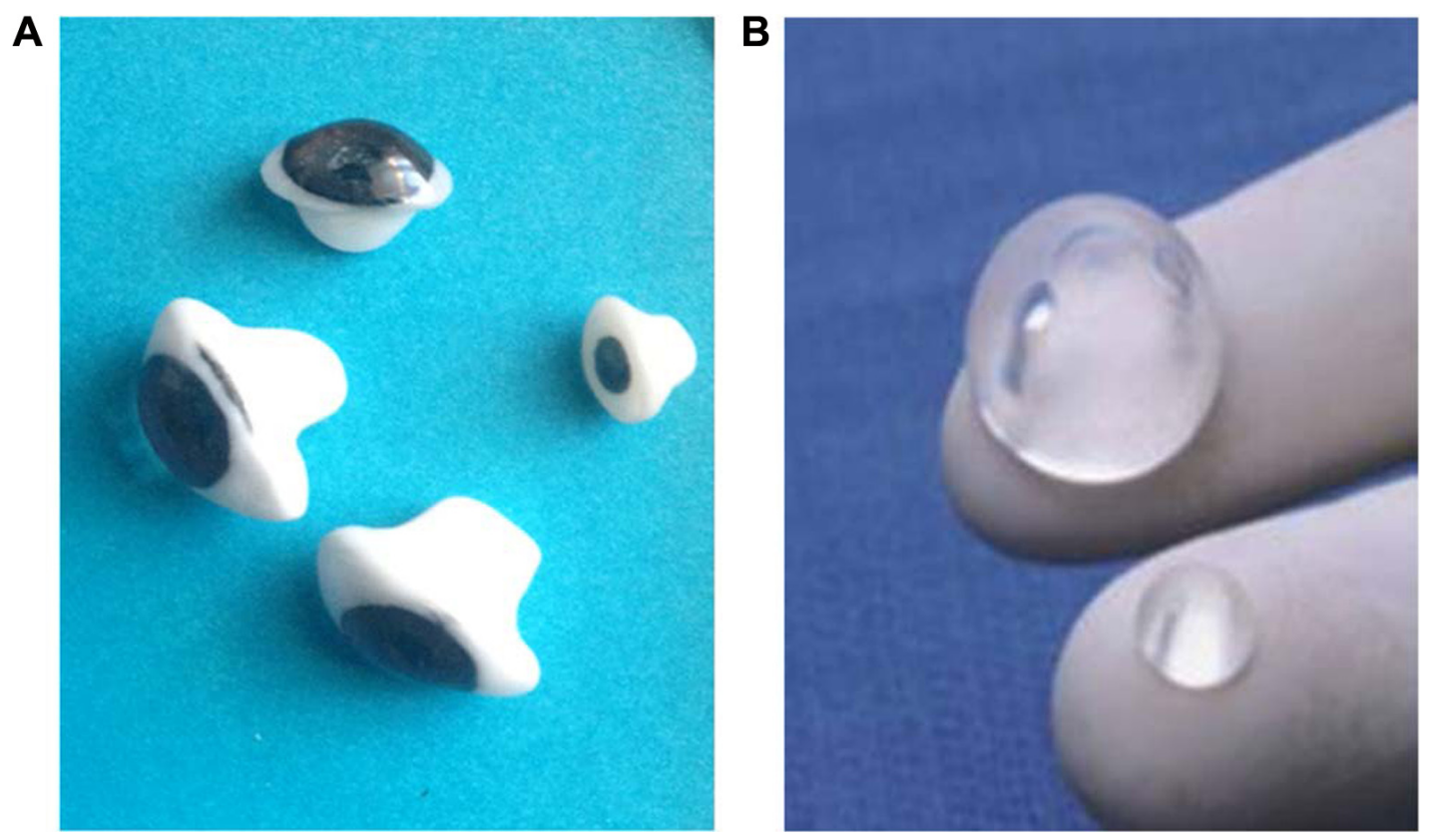

Figure I (A) Specially designed “acorn-shaped" prosthesis and (B) expandable hydrogel socket implants. 
was determined by measuring the horizontal eyelid length (the distance between the medial and lateral canthus in $\mathrm{mm}$ ) by a ruler, and the volume of the last prosthesis by submerging it in a calibrated cylinder filled with water and recording the volume in $\mathrm{cm}^{3}$ of water displaced by the prosthesis. No hospitalization or general anesthesia was required for any of our patients, but occasionally during the serial fitting of these infants chloral hydrate $(100 \mathrm{mg} / \mathrm{kg})$ was used for sedation. Approval of the ethical committee of Cairo University Specialized Pediatric Hospital Ophthalmology Department was obtained and written informed consents were obtained from parents or legal guardians.

\section{Results}

At the age of 2 years, the mean horizontal eyelid length increased from a mean of $11.6 \pm 4.5$ at the beginning of treatment to $19.4 \pm 4.6 \mathrm{~mm}$ and the volume of the expander from a mean of $0.6 \pm 0.2$ to $2.0 \pm 0.3 \mathrm{~cm}^{3}$. Apart from transient occasional conjunctival infection in a few cases (five) requiring removal of the prosthesis for a few days and application of antibiotic drops and ointment, no serious complications were encountered throughout the follow-up period that ranged from 10 months to 4.5 years (mean $=23$ months). Eight of the children became eligible for further cosmetic improvement by lid surgery or dermis fat orbital reconstruction. Table 1 shows the descriptive

Table I Data of all anophthalmic eyes

\begin{tabular}{|c|c|c|c|c|c|c|c|}
\hline Case & $\begin{array}{l}\text { Age } \\
\text { (weeks) }\end{array}$ & Pre-HL & Post-HL & HL diff & Pre-V & Post-V & V diff \\
\hline I & 12 & 10 & 20 & 10 & 0.6 & 1.8 & 1.2 \\
\hline 2 & 6 & 9 & 18 & 9 & 0.4 & 1.5 & I.I \\
\hline 3 & 48 & 16 & 25 & 9 & 0.9 & 2.2 & 1.3 \\
\hline 4 & 48 & 18 & 26 & 8 & 0.9 & 2.3 & I.4 \\
\hline 5 & 6 & 9 & 16 & 7 & 0.6 & 1.7 & I.I \\
\hline 6 & 6 & 9 & 20 & II & 0.4 & 1.6 & 1.2 \\
\hline 7 & 6 & 12 & 16 & 4 & 0.7 & 2.1 & I.4 \\
\hline 8 & 18 & 14 & 20 & 6 & 0.8 & 2.4 & 1.6 \\
\hline 9 & 20 & 16 & 22 & 6 & 0.6 & 1.7 & I.I \\
\hline 10 & 6 & 8 & 14 & 6 & 0.4 & 1.8 & 1.4 \\
\hline II & 6 & 8 & 14 & 6 & 0.4 & 2 & 1.6 \\
\hline 12 & 8 & 16 & 27 & II & 0.4 & 2.1 & 1.7 \\
\hline 13 & 4 & 5 & 12 & 7 & 0.4 & 1.8 & $\mathrm{I} .4$ \\
\hline 14 & 4 & 6 & 16 & 10 & 0.4 & 2.2 & 1.8 \\
\hline 15 & 10 & 8 & 18 & 10 & 0.8 & 2 & 1.2 \\
\hline 16 & 14 & 14 & 20 & 6 & 0.7 & 2 & 1.3 \\
\hline 17 & 60 & 20 & 26 & 6 & 0.9 & 2.4 & 1.5 \\
\hline Mean & 16.6 & 11.6 & 19.4 & 7.8 & 0.6 & 2 & 1.4 \\
\hline SD & 17.7 & 4.5 & 4.6 & 2.1 & 0.2 & 0.3 & 0.2 \\
\hline
\end{tabular}

Abbreviations: Pre-HL, pretreatment horizontal eyelids length in $\mathrm{mm}$; Post-HL, posttreatment horizontal eyelids length in $\mathrm{mm}$; $\mathrm{HL}$ diff, the difference in the horizontal eyelids length from the start of treatment until the age of 2 years in mm; Pre-V, pretreatment socket volume in $\mathrm{mL}$; Post-V, posttreatment socket volume in $\mathrm{mL}$; $\mathrm{V}$ diff, the difference in the socket volume from the start of treatment until the age of 2 years in $\mathrm{mL}$; SD, standard deviation. data of all eyes. Figures 2 and 3 show the photos of some cases throughout the socket expansion process.

\section{Discussion}

The term clinical anophthalmia is used to describe cases in which no globe can be identified by clinical examination and also includes cases of severe microphthalmia. Both anomalies occur usually due to early insults during the second stage of development (first 4-8 weeks gestation) resulting in failure of proper development of the optic vesicle. The developmental defect is located in Pfeifer's diencephalic region. This region is defined as the bilateral zone between the frontonasal and the posterolateral regions of the face and stretches from the temples to the upper lip crossing the orbits and the lateral parts of the nose. ${ }^{12}$ It is therefore not uncommon to find other craniofacial anomalies of this region, such as hemifacial atrophy, cleft lip, and cleft palate associated with each other; this also explains the heterogeneity of the clinical picture of anophthalmia.

The management of congenital anophthalmos is a long-term commitment for the family, ocularist, and ophthalmologist. Each case is unique regarding its phenotypic presentation and associated anomalies, the severity of orbital and adnexal constriction, the age of initial therapy, and even in the response to treatment (rate of growth). The goal is to rehabilitate the socket in order to allow the retention of a suitable prosthesis and develop an acceptable appearance or symmetry. This implies achieving adequate lid levels and contours, sufficiently deep conjunctival fornices, and orbital volume augmentation. But, normal lid size and excursion as well as full versions of the prosthesis are unattainable and frank discussion with the family is very important to avoid both unrealistic expectations and overall loss of hope from any outcome of therapy. The technique described earlier has been found valid and successful for our management goals.

The ideal conformer or socket expander should follow the contour of the socket and fill the fornix without excessive stretching or pressure on the conjunctiva and tenon and allow the child to close the eyelids easily over the conformer. In this work, two types of socket expanders were used: specially designed case-tailored acrylic solid conformers (used mainly) and hydrogel expandable expanders (used occasionally, especially at the beginning of therapy).

The idea of this specially designed solid acrylic conformer used came from the finding that the congenital anophthalmic socket is usually "acorn-like" in shape having a depression or pit at the apex of the socket. ${ }^{11}$ This configuration is put into consideration either in custom-made impressions or 

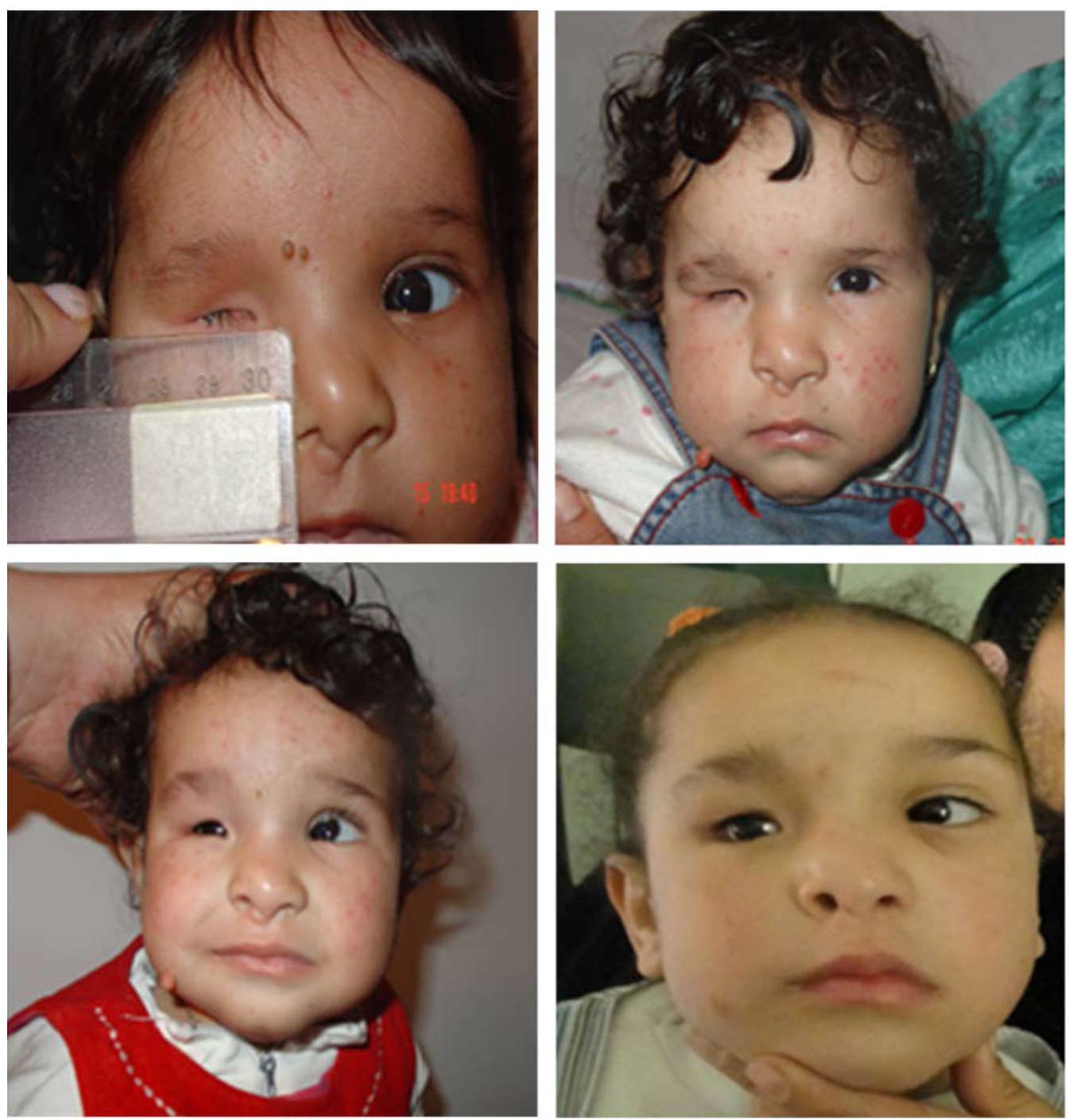

Figure 2 Stepwise socket expansion in a patient with unilateral anophthalmia.

molds taken under general anesthesia or in those special designs used (Figure 1A). During the initial and subsequent examination of the socket, one is able to identify and draw its shape, depth, and the position of the pit. This is incorporated in designing semi-custom-made conformers. The size of this initial fit allows the ocularist to design a sequence of progressively larger conformers for the next stages. The advantages of this special design are 1) it acts as both a conformer and
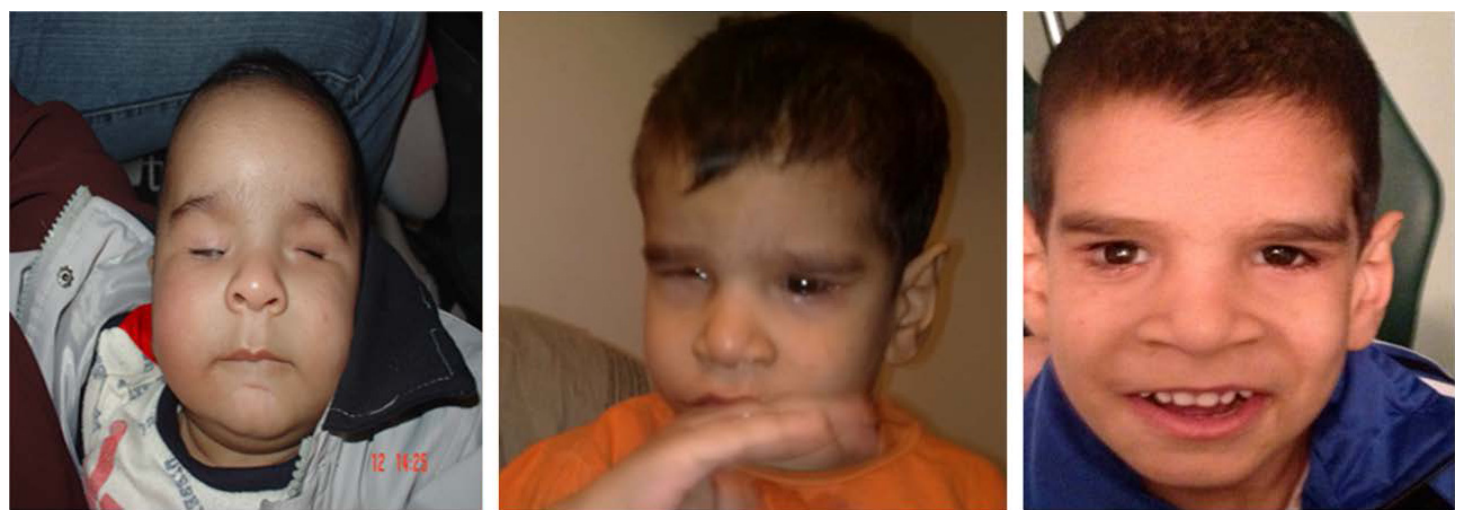

Figure 3 Stepwise socket expansion in a boy with bilateral clinical anophthalmia. 
prosthesis, 2) it is possible to fit in an ambulatory manner, 3 ) it gives better stability than regular thin conformers as it properly fits in the configuration of the socket thus reducing the risk of frequent rotation and expulsion, 4) it expands the socket in the anteroposterior as well as the horizontal and vertical components, 5) it does not need suturing the eyelids even in some cases of deficient eyelid support due to coloboma, 6) it is easier to fit by the caregiver, and 7) unlike molded shapes, there is a minimal space between the conformer and the socket allowing for drainage of any secretions and the application of topical eye drops.

With the introduction of self-inflating hydrogen copolymers of methyl-methacrylate and $\mathrm{N}$-vinylpyrrolidon by Wiese et al, ${ }^{13}$ the concept of using osmosis to induce a gradual swelling of a special hydrogel has been rapidly adopted by many ophthalmologists to induce a gradual socket expansion. ${ }^{5,14,15}$ The expanders are placed in their dry, contracted states, and expand gradually to their full size via osmosis of surrounding tissue fluid, with up to a tenfold increase in volume, so, for example, the $0.9 \mathrm{~mL}$ hydrogel implant mentioned in this study has an initial volume of $0.13 \mathrm{~mL}$.

Suturing of the eyelids, until the implant swells enough to be retained in the orbit, exposes the child to the complications of general anesthesia as well as suture complications, such as infection or cheese wiring. Therefore, in this study cyanoacrylate glue (Histoacryl $^{\circledR}$, TissueSeal LLC) was used instead. Cyanoacrylate glues have been used successfully for corneal conditions with a good safety profile. It has been also used for skin closure in pediatric lid lacerations, for temporary tarsorrhaphy in case of corneal exposure and amblyopia treatment, and recently as an alternative to suture in pediatric socket expansion. ${ }^{8,15,16}$

Many of these children have associated eyelid anomalies with individual variations, such as blepharophimosis, eyelid coloboma, and ptosis. The results of early surgery on the lids have not been impressive. In fact, even a procedure as simple as a lateral canthotomy, a common first procedure performed on many of our referred patients, can cause healing with fibrosis and further contractures and the loss of an important point of capture for the prosthesis throughout growth and development. Hence, a judicious and conservative approach to such ancillary lid procedures is advisable and better postponed after the first 2 years of age or more. The technique described worked well with a case of eyelid coloboma involving a quarter of the upper eyelid without prior correction. With continued follow-up beyond the age of 3 , eight of our patients were treated by lateral or medial canthoplasty to facilitate prosthetic retention, repair of eyelid coloboma, or dermis fat grafts. Several of our patients who appeared to have ptosis at the beginning of treatment had the ptotic appearance of the eyelid improved and their lid elevated as they responded to serial socket expansion or later to orbital implantation. Therefore, early ptosis surgery is not advisable until long-term stabilization is observed, and, if needed, frontalis suspension was performed so as not to compromise the superior fornix and prosthetic retention if a levator muscle surgery was attempted.

This study did not aim to compare between solid and hydrophilic expanders; this is not possible because the number of hydrogel expanders used were relatively few and in most cases were followed by larger specially designed prostheses when the sockets expanded.

As in most published studies dealing with this subject, one of the defects of this study is that there is no control group. Comparison with a control group is difficult unless a really huge number of cases are included and this is not easy given the rarity of the condition and the long-term follow-up required. Hardly any randomized trials have been published in this field, and thus clinical decision making is usually based on the results of case series and observational studies.

\section{Conclusion}

Successful increase in the horizontal eyelid length as well as the conjunctival socket volume in anophthalmic infants could be achieved by a simple outpatient procedure without the need for hospitalization or general anesthesia or lid suturing. This technique improves the compliance of the parents, encourages earlier rehabilitation and, needless to say, saves time, cost, and effort. It allows retention of prosthesis in children below 2 years thus obviating the need of early interventional surgery, which is better deferred to a later stage if necessary.

\section{Acknowledgments}

The author is grateful to Ahmed Ibrahim, the talented ocularist (a eyes ahmed@yahoo.com) who has converted the socket drawings into specially designed real conformers and prostheses and who has always managed to help a lot of anophthalmic children with patience and enthusiasm. And, also, to the families of the children for their dedication and commitment and for allowing the use of the pictures of their children without hiding their identity to help show the effect of treatment.

The results of this study were presented in part in the American Association for Pediatric Ophthalmology and Strabismus (AAPOS) meeting 2013 as an oral presentation. An abstract was published in the "AAPOS 2013 Meeting Abstracts" (http://www.jaapos.org/issue/S1091-8531(13) $\underline{\mathrm{X} 0002-3})$. 


\section{Disclosure}

The authors have no financial interests to disclose. The authors report no conflicts of interest in this work.

\section{References}

1. Shah SP, Taylor AE, Sowden JC, et al; Surveillance of Eye Anomalies (SEA-UK) Special Interest Group. Anophthalmos, microphthalmos, and typical coloboma in the United Kingdom: a prospective study of incidence and risk. Invest Ophthalmol Vis Sci. 2011;52(1):558-564.

2. Tucker SM, Sapp N, Collin R. Orbital expansion of the congenitally anophthalmic socket. Br J Ophthalmol. 1995;79(7):667-671.

3. Yang G, Wang J, Chang Q, Wang Z, Geng Y, Li D. Digital evaluation of orbital development in Chinese children with congenital microphthalmia. Am J Ophthalmol. 2012;154(3):601-609.

4. Tse DT, Abdulhafez M, Orozco MA, Tse JD, Azab AO, Pinchuk L. Evaluation of an integrated orbital tissue expander in congenital anophthalmos: report of preliminary clinical experience. Am JOphthalmol. 2011;151(3):470-482.

5. Mazzoli RA, Raymond WR 4th, Ainbinder DJ, Hansen EA. Use of selfexpanding, hydrophilic osmotic expanders (hydrogel) in the reconstruction of congenital clinical anophthalmos. Curr Opin Ophthalmol. 2004;15(5): 426-431.

6. Wolfe SA. Microphthalmos. In: Wolfe SA, Berkowitz S, editors. Plastic Surgery of the Facial Skeleton. Boston: Little, Brown \& Co; 1989;670.
7. Gundlach KK, Guthoff RF, Hingst VH, Schittkowski MP, Bier UC. Expansion of the socket and orbit for congenital clinical anophthalmia. Plast Reconstr Surg. 2005;116(5):1214-1222.

8. Quaranta-Leoni FM. Congenital anophthalmia: current concepts in management. Curr Opin Ophthalmol. 2011;22(5):380-384.

9. Kataev MG, Filatova IA, Verigo EN, Kiriukhina SL. Potentialities of conservative and surgical treatment of patients with congenital microphthalmia and anophthalmia. Vestn Oftalmol. 2000;116(6):9-13.

10. Wagner A, Schneider C, Lagogiannis G, Hollman K. Pulsatile expansion therapy for orbital enlargement. Int J Oral Maxillofac Surg. 2000; 29(2):91-95.

11. Merritt JH, Trawnik WR. Prosthetic and surgical management of congenital anophthalmia. J Ophthalmic Prosthet. 1997;2:1-14.

12. Gundlach KK, Pfeifer, G. Classification of facial malformations. Int $J$ Oral Surg. 1981;10(Suppl 1):267-272.

13. Wiese KG, Vogel M, Guthoff R, Gundlach KK. Treatment of congenital anophthalmos with self-inflating polymer expanders: a new method. J Craniomaxillofac Surg. 1999;27(2):72-76.

14. Schittkowski MP, Gundlach KK, Guthoff RF. Treatment of congenital clinical anophthalmos with high hydrophilic hydrogel expanders. Ophthalmologe. 2003;100(7):525-534.

15. Vera L, Benzerroug M, Gueudry J, et al. An update on the use of tissue adhesives in ophthalmology. J Fr Ophtalmol. 2009;32(4):290-305.

16. Trivedi D, McCalla M, Squires Z, Parulekar M. Use of cyanoacrylate glue for temporary tarsorrhaphy in children. Ophthal Plast Reconstr Surg. 2014;30(1):60-63.
Clinical Ophthalmology

\section{Publish your work in this journal}

Clinical Ophthalmology is an international, peer-reviewed journal covering all subspecialties within ophthalmology. Key topics include: Optometry; Visual science; Pharmacology and drug therapy in eye diseases; Basic Sciences; Primary and Secondary eye care; Patient Safety and Quality of Care Improvements. This journal is indexed on Submit your manuscript here: http://www.dovepress.com/clinical-ophthalmology-journal

\section{Dovepress}

PubMed Central and CAS, and is the official journal of The Society of Clinical Ophthalmology (SCO). The manuscript management system is completely online and includes a very quick and fair peer-review system, which is all easy to use. Visit http://www.dovepress.com/ testimonials.php to read real quotes from published authors. 